\title{
Retinal Growth in Foveated Teleosts: Nasotemporal Asymmetry Keeps the Fovea in Temporal Retina
}

\author{
Stephen S. Easter, Jr. \\ Department of Biology, University of Michigan, Ann Arbor, Michigan 48109-1048 and Vision, Touch, and Hearing Research \\ Centre, Department of Physiology and Pharmacology, University of Queensland, Brisbane, QLD 4067, Australia
}

Fish retinas continue to grow throughout life by adding neurons at the margin, with the result that cells born at a peripheral site are steadily displaced toward the center of the enlarging retina. This presents a functional problem for fish with specialized temporal areas such as a fovea-how to reconcile continual growth with the maintenance of a temporal location for the fovea. One possibility is that the retina grows asymmetrically, with most new retina added nasally, relatively little temporally.

I have tested this hypothesis by evaluating retinal growth in marine teleosts from 15 families, both foveated and unfoveated. The pattern of growth was revealed by exploiting the fact that each new generation of ganglion cells sends its axons into the optic nerve as a cohort; small grains of the carbocyanine dye $1,1^{\prime}$-dioctadecyl-3,3,3',3'-tetramethylindocarbocyanine were applied to various sites in the cross section of the optic nerves of adults, and the retrogradely labeled cell bodies in the retina were visualized in wholemounts. The labeled cells lay in annuli, each one a generation of ganglion cells.

Representatives of seven of the families showed clearly asymmetric growth: the labeled annuli were close together on the temporal side and more distant nasally, the embryonic fissure curved from its ventral origin toward the temporal side, and in six of these families, labeled fibers from temporal retina skirted the fovea. Members of the other eight families, without specialized areas, had more symmetric retinal growth: labeled annuli were equally spaced on all sides, the embryonic fissure was vertical, and there were no skirting fibers.

The following hypothesis is supported: the retina grows asymmetrically, and maintains the area for acute vision oriented toward the anterior field.

The retina of a teleost fish grows throughout much of the animal's life (Muller, 1952; Johns and Easter, 1977; Meyer, 1978). The enlargement is both passive, as the retina is stretched by

\footnotetext{
Received Dec. 4, 1991; accepted Jan. 16, 1992.

This work was carried out while on sabbatical leave at the Vision, Touch, and Hearing Centre, with support from the Australian Research Council and NIH Research Grant EY-00168. I thank Prof. Jack Pettigrew, Dr. David Vaney, and others at the Centre for their help and hospitality. I thank Ms. Rita Collins and Celeste Malinoski for doing the histology, and Drs. David Cameron, Peter Hitchcock, Jack Pettigrew, Pamela Raymond, John Scholes, Steve Wilson, Ms. Riva Marcus, and Mr. John Burrill for their comments on the manuscript.

Correspondence should be addressed to Stephen S. Easter, Jr., Department of Biology, University of Michigan, Ann Arbor, MI 48109-1048.

Copyright (C) 1992 Society for Neuroscience $0270-6474 / 92 / 122381-12 \$ 05.00 / 0$
}

the inflation of the eye, and active, as new cells are added. Rods are added interstitially throughout the retina (Johns and Fernald, 1981; Raymond and Rivlin, 1987), but all the other cell types are added at the margin, where stem cells proliferate to produce more stem cells and postmitotic neuroblasts (Muller, 1952; Hollyfield, 1972; Johns, 1977). The stem cells continue to proliferate on the margin; the postmitotic cells remain just inside it, and differentiate into the various types of retinal neuron. The new neurons thus form an annulus around the edge of the eye, central to the marginal germinal zone from which they recently emerged, and peripheral to the preceding generation of neurons (Scholes, 1979; Rusoff and Easter, 1980).

Such appositional addition of new annuli provides an interesting complication for vision. In goldfish, for example, the eyes of large and small fish are conformal; in both, the retina subtends the same visual angle, about half of a hemisphere, and the optic disk lies approximately at the center (Easter et al., 1977). These facts, coupled with the annular mode of cell addition, imply that as the eye grows, the receptive fields of retinal neurons must continually move from the periphery toward the center of the retina's visual field. For instance, a ganglion cell born on the temporal margin would initially have its receptive field in front of the fish, but as new cells are added to the margin, this same cell's receptive field would shift laterally and posteriorly. How the brain manages this ever-changing relationship with the outside world is completely unknown, but the problem is slightly simplified by the fact that the goldfish retina lacks a specialized area; that is, a region of high cell density (RHCD) (Johns and Easter, 1977). Because cell density varies relatively little with position, one part of the retina is probably functionally equivalent to another, and the retinal modules that are added at the edge resemble those everywhere else in the retina.

The problem of reconciling annular growth with vision is more complicated in those fish with an RHCD. Walls (1942, p 304) lists 24 teleost species, from 13 genera, that have a fovea (an RHCD marked by a pit in the inner limiting membrane), and notes that many others have areae that are RHCDs not associated with a pit (for examples, see Collin and Pettigrew, 1988a,b). Presumably these RHCDs-fovea or area-are specialized for high resolution (Collin and Pettigrew, 1989), as in the human eye. The fish fovea differs structurally from the human fovea, however, in that it has all the retinal layers present except that of the optic fibers. The ganglion cell layer lies at the bottom of the pit, the walls of which are formed by the mounded optic axons.

In almost all fish that have one, the RHCD is in far temporal retina, and this location is vulnerable to the effects of annular growth. How can a temporal RHCD remain temporal, and avoid 
Table 1. Types of fish examined in this study, grouped according to whether the retina grew symmetrically or asymmetrically (see Results)

Symmetric growth

1. Acanthuridae (surgeon fish)

a. Paracanthurus hepatus (blue tang) (1)

2. Blenniidae (blennies)

a. Salarius sinuosus (fringelip blenny) (2)

b. Istiblennius edentula (rippled rockskipper) (2)

3. Chaetodontidae (butterfly fish and coral fish)

a. Chelmon rostratus (beaked coral fish) (2)

4. Gobiidae (gobies)

a. Bathygobius fuscus (common goby) (2)

5. Mullidae (goatfish)

a. Upeneus tragula (freckled goatfish) (2)

6. Opistognathidae (jawfish)

a. Unidentified (1)

7. Pomacentridae (damselfish)

a. Dascyllus aruanus (humbug dascyllus) (1)

b. Chrysiptera unimaculata (onc-spot damsclfish) (1)

8. Syngnathidae (pipe fish and sea horses)

a. Trachyrhamphus bioarctatus (short-tailed pipefish) (1)

b. Hippocampus kuda (spotted sea horse) (1)

c. Unidentified (sea horse) (1)

Asymmetric growth

1. Callionymidae (dragonets)

a. Unidentified (3)

2. Cirrhitidae (hawkfish)

a. Cirrhitichthys falco (dwarf hawkfish) (1)

3. Labridae (wrasses)

a. Macropharygodon negrosensis (black wrasse) (1)

4. Monacanthidae (leatherjackets)

a. Unidentified (8)

5. Pinguipedidae (sand perch)

a. Parapercis cylindrica (sharpnose sand perch) (4)

b. Parapercis nebulosa (barred sand perch) (10)

6. Platycephalidae (flatheads)

a. Unidentified (3)

7. Tetraodontidae (puffer fish)

a. Canthigaster valentini (black saddled toby) (1)

b. Unidentified (1)

Each numbered row gives the family, followed by the common name in parentheses. Each lettered row gives the genus and species, with common name in parentheses, followed by the number of such fish examined. "Unidentified" means that the fish could only be identified by family; genus and species are unknown.

drifting to the center of the retina as the retina enlarges? Eye migration could help; if the RHCD drifted centrally, and the eyes migrated frontally, the RHCD would continue to look forward. But in most fish species (flatfish excepted), the eyes do not migrate-they begin and remain lateral, with typically $40^{\circ}$ of frontal binocular overlap. Another possible solution to the problem is for the retina to grow asymmetrically. If the RHCD were formed in temporal retina early in life, and relatively little retina were subsequently added temporally, relative to the other quadrants, then the receptive field of the specialized area would remain anterior. Other possible solutions will be presented in the Discussion.

I have investigated retinal growth in marine fishes with and without an RHCD. Several generations (annuli) of ganglion celis were labeled in individual retinas, and, from retinal whole mounts, the pattern of growth was inferred.

A preliminary account has been published as an abstract (Easter, 1991).

\section{Materials and Methods}

In fish, each generation of ganglion cells sends its axons in a common fascicle in the optic nerve (Scholes, 1979; Rusoff and Easter, 1980; Easter et al., 1981), so if a small quantity of retrograde tracer is applied to a fascicle, an annulus of ganglion cells is labeled (Rusoff and Easter, 1981). Most of the fish described below had ribbon-shaped optic nerves (Tapp, 1973; Anders and Hibbard, 1974), in which sequential generations of axons are laid out linearly along the ribbon, oldest at the central end, youngest at the peripheral (ventral) end (Scholes, 1979). Thus, it was possible to apply traccr at a particular site in the cross section of the nerve and predict the approximate retinal location of the labeled cells.

Fish were obtained by netting at the Heron Island Research Station on the Great Barrier Reef, in a trawl on Moreton Bay, and by purchase from a local pet store. Individual fish were anesthetized by immersion in a $0.1 \%$ solution of tricaine methane sulfonate, and after the aorta was cut, both eyes were removed with a stump of the optic nerve attached. The cornea, lens, and iris were removed, and the fish's standard length and lens diameter were recorded. Both eye cups were fixed by immersion in $2 \%$ or $4 \%$ paraformaldehyde in $0.1 \mathrm{M}$ phosphate buffer at room temperature for $4 \mathrm{hr}$ to 2 weeks. The dye 1,1'-dioctadecyl3,3,3',3'-tetramethylindocarbocyanine (DiI; obtained from Molecular Probes) (Honig and Hume, 1986), which diffuses in the plasma membrane in fixed tissue (Godement et al., 1987), was applied to one or more sites on the optic ncrve stumps, or intraretinally, and the tissue was returned to fix at $37^{\circ} \mathrm{C}$. After a suitable interval $(4 \mathrm{~d}$ to 2 weeks, determined by examining the intact tissue in a fluorescence microscope), the tissue was prepared for whole-mounting. The retina and adherent pigmented epithelium were dissected away as a unit from the rest of the eye, relaxing cuts were made to allow it to lie flat, and it was mounted with the inner limiting membrane up, in $50 \%$ phosphate buffer, $50 \%$ glycerol, and coverslipped. The intersection of the embryonic fissure with the margin was taken as the ventral pole, and all whole-mounts are shown with the ventral pole below, the dorsal pole up, and the nasotemporal axis horizontal.

Some eyes were embedded in glycomethacrylate, sectioned, and stained with toluidine blue.

Slides were viewed and photographed on a Zeiss Universal compound microscope with rhodaminc-cube epiillumination.

Table 1 lists the 15 teleost families, and where possible the genera and species, included in this study. Some were selected because they belonged to families that had been reported to include species with RHCDs (blennies, wrasses, sand perches, and damselfish: Collin, 1987; Collin and Pettigrew, 1988a,b; pipefish and puffer fish: Kahmann, 1934). Others were chosen because they made frequent omnidirectional saccadic eye movements, which Walls (1942) has suggested is associated with a specialized area (hawkfish and leatherjackets: S. S. Easter, unpublished observations). The others were used to enlarge the sample. Species identifications were made according to Randall et al. (1990).

\section{Results}

\section{Symmetric growth}

This pattern of growth is treated first because it introduces the data most conveniently. Figure 1 shows a whole-mounted left retina from a butterflyfish. The optic papilla is a disk-shaped structure at the approximate center of the retina, into which a large number of fluorescent axons converge from several concentric annuli of labeled somata. The distance from the papilla to the margin varies, being smallest in the temporal retina and largest nasally, but the differences are not great.

Some of the annuli in Figure 1 are incomplete. Specifically, five of them (2-6) are evident on all of the nasal hemiretina and on much of the temporal half. The innermost (1) is very faint temporally, and four more peripheral ones (7-I0) are visible only in the dorsotemporal quadrant. Incomplete annuli were noted in nearly every whole-mount. The missing scctors seemed to be randomly distributed, and were assumed to result from incomplete labeling of the fascicles rather than gaps in the proliferative zone or selective cell death after proliferation.

This retina had grown symmetrically, as the space between any pair of annuli is roughly the same at all positions along their arcs. The optic papilla is linked to the ventral pole of the retina by the embryonic fissure, which is patent, as in most fish. The fissure's intersection with a labeled annulus marks the location 
The Journal of Neuroscience, June 1992, 12(6) 2383

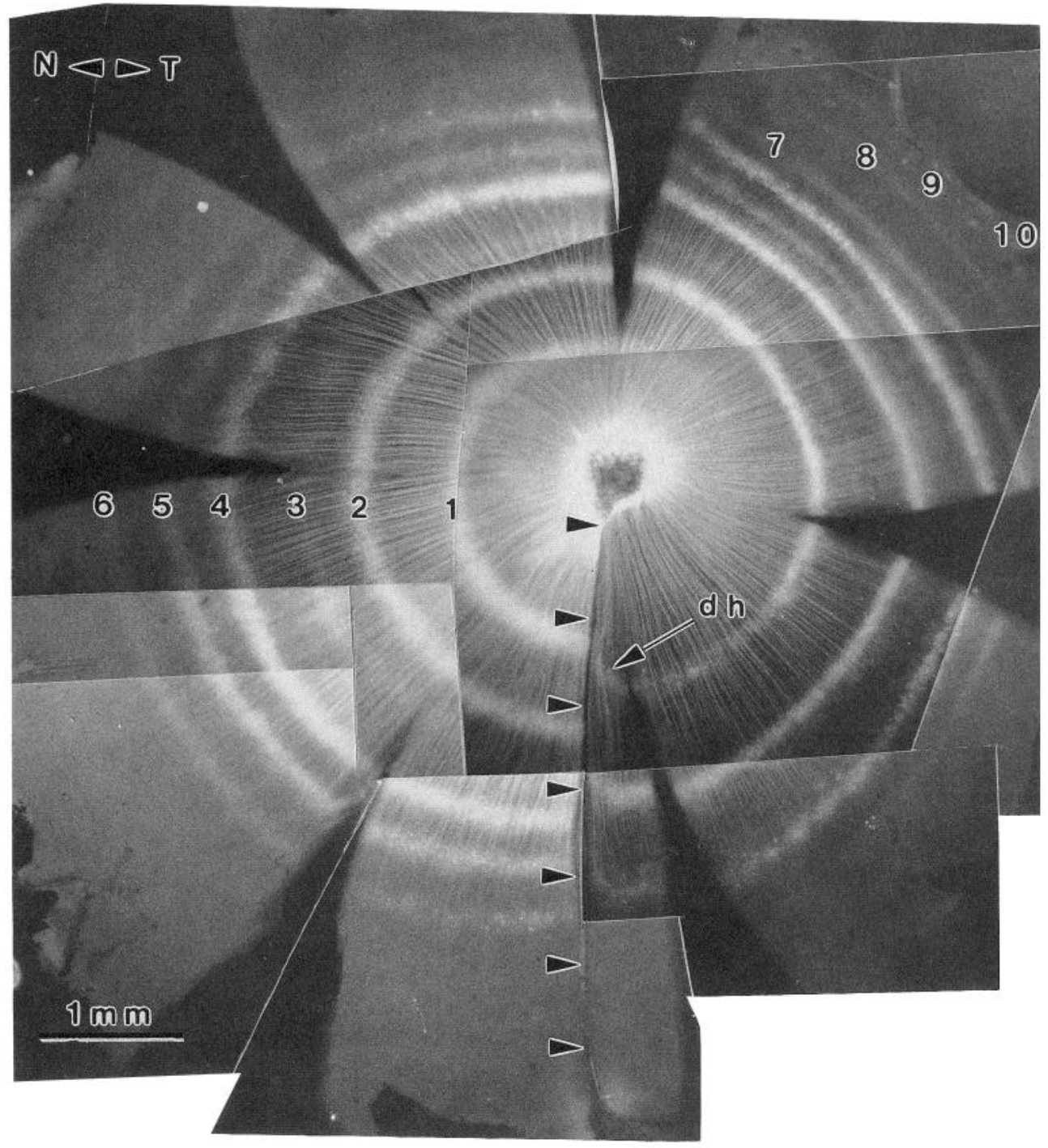

Figure 1. Left retinal whole-mount of Chelmon rostratus (Chaetodontidae) illustrating symmetric retinal growth. The numbers $1-10$ indicate the 10 annuli (some incomplete) of ganglion cell somata labeled retrogradely through the optic nerve. The arrowheads indicate the embryonic fissure extending from the optic papilla to the ventral pole of the retina. The nasal and temporal directions are given by $N$ and $T$, respectively, in this and all other wholemounts. The dorsal hook of the second annulus is indicated by $d h$. of the ventral pole of the retina at the time when that annulus lay at the retinal margin. The viewer expects to see complete circles, but in fact, individual annuli intersect the fissure at slightly disparate locations from the nasal and temporal sides. This is because the temporal limb of the annulus hooks sharply dorsalward near the fissure. A similar hook was seen at the ventral pole in retinas in which the outermost annulus of cells was labeled (Fig. 2), so the dorsal hooks of Figure 1 are presumed to be relics of the time when the labeled annulus lay at the margin.

Fish from four other families (surgeonfish, gobies, jawfish, and damselfish) showed essentially similar labeling; that is, a disk-shaped optic papilla, radially symmetric annuli, radially convergent axons, and a vertical embryonic fissure. Three others (blennies, goatfish, and pipefish) had roughly symmetric annuli,

Figure 2. Right retinal whole-mount of Istiblennius edentula (Blenniidae) illustrating the dorsal hook $(d h)$ at the retinal margin. Arrowheads show the embryonic fissure; $T$ and $N$, the temporal and nasal directions. The outermost annulus of cells was labeled.

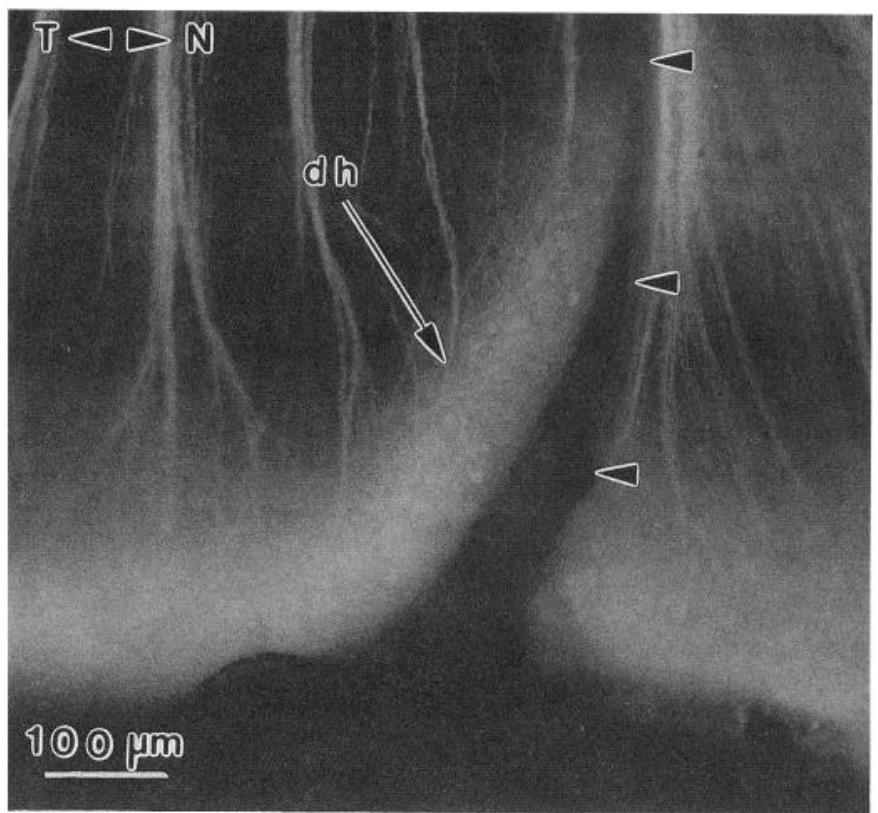




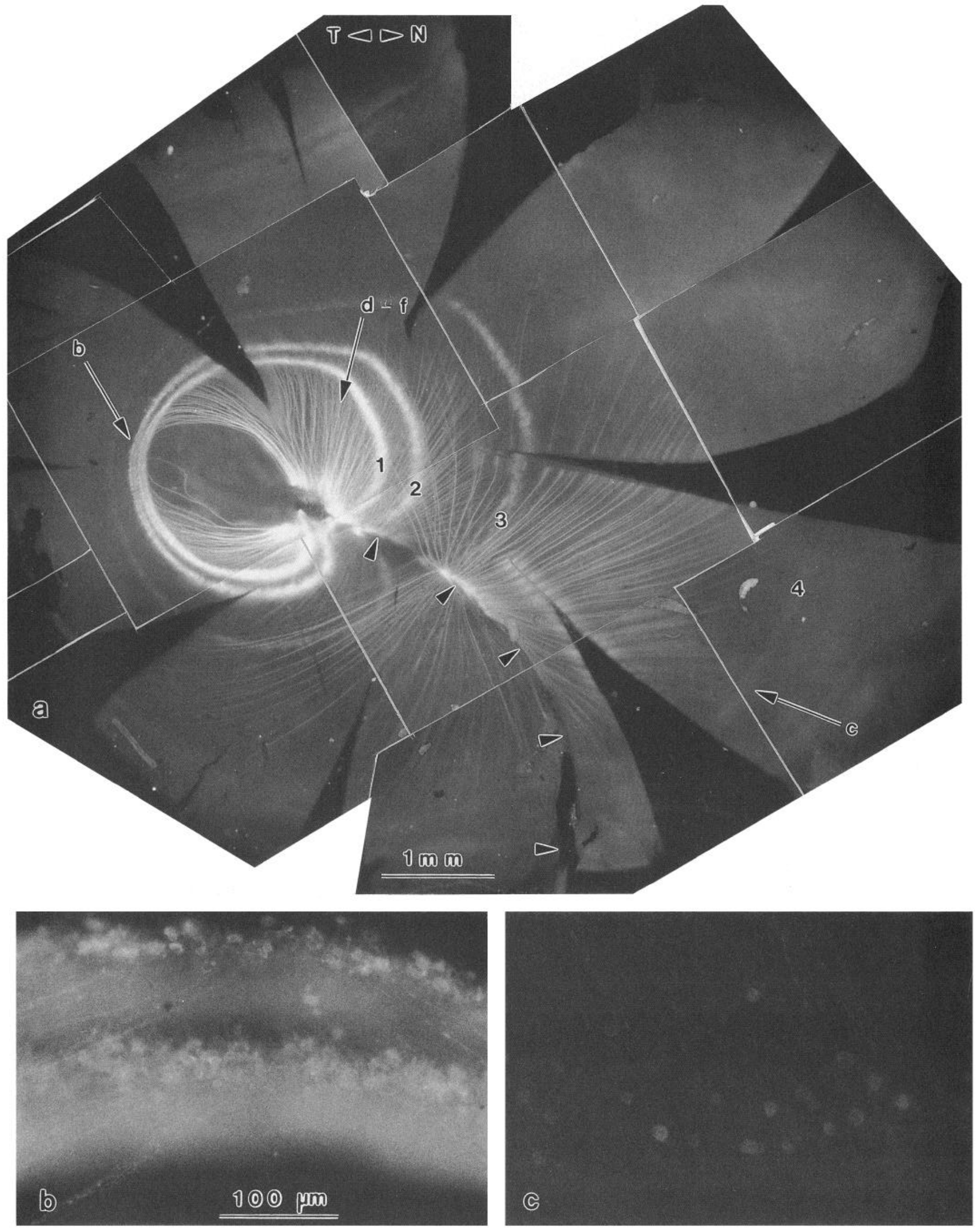




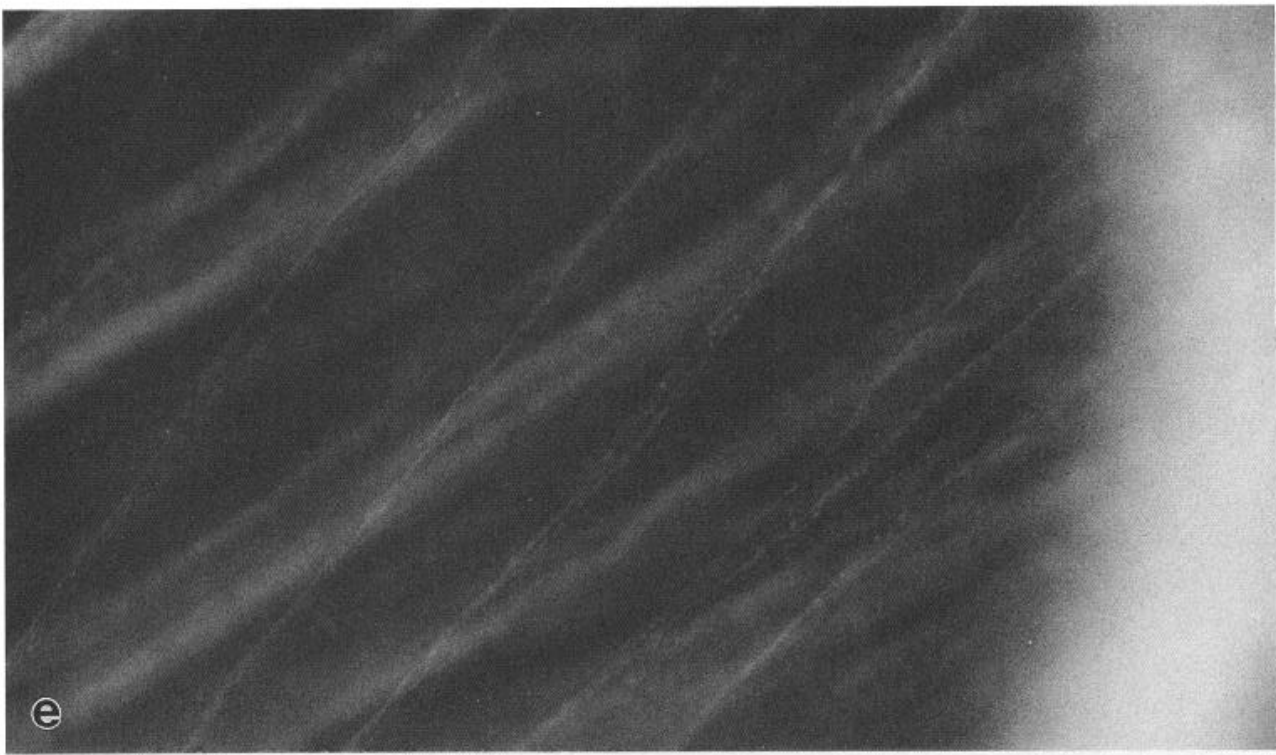

Figure 3. Right retinal whole-mount of Parapercis cylindrica (Pinguipedidae) illustrating asymmetric retinal growth. $a$, Four annuli (1-4) are evident, although the fourth is very faint.

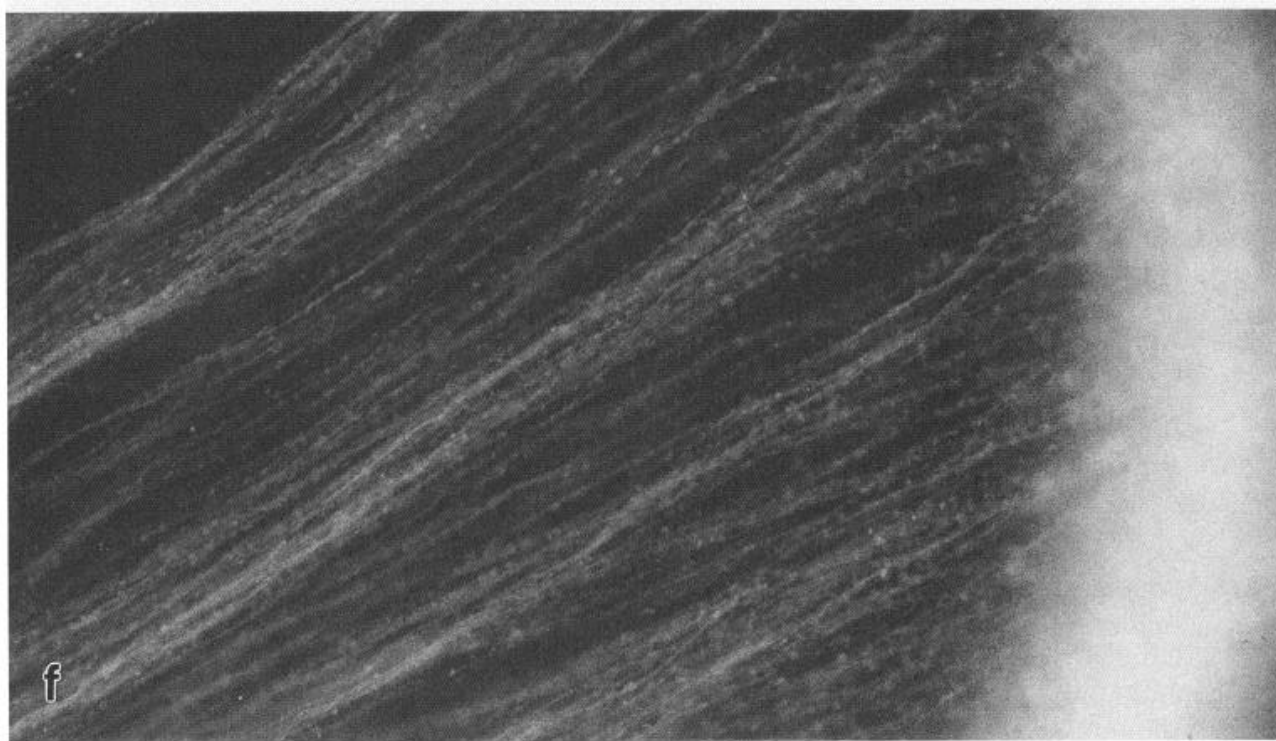

Four populations of axons converge on the elongated optic papilla that extends through much of the embryonic fissure, which is indicated by arrowheads. An axon-free region inside the centralmost annulus includes the fovea. The letters $b-f$ indicate the regions shown in the more highly magnified pictures in those panels. $b$, The two groups of labeled somata (in focus) are separate from each other, and each is underlain by labeled dendrites (out of focus). $c$, A segment of the fourth annulus shows that the cells in nasoventral retina are in lower density than in dorsotemporal retina. $d-f$, Three views, at progressively deeper planes, of the same field, to show the stratification of the axons. The bright contour to the right in each photo is the first annulus $(a)$. The more peripheral (younger) somata send their axons more superficially than their predecessors. Scale bar in $b$ applies to $b-f$. 

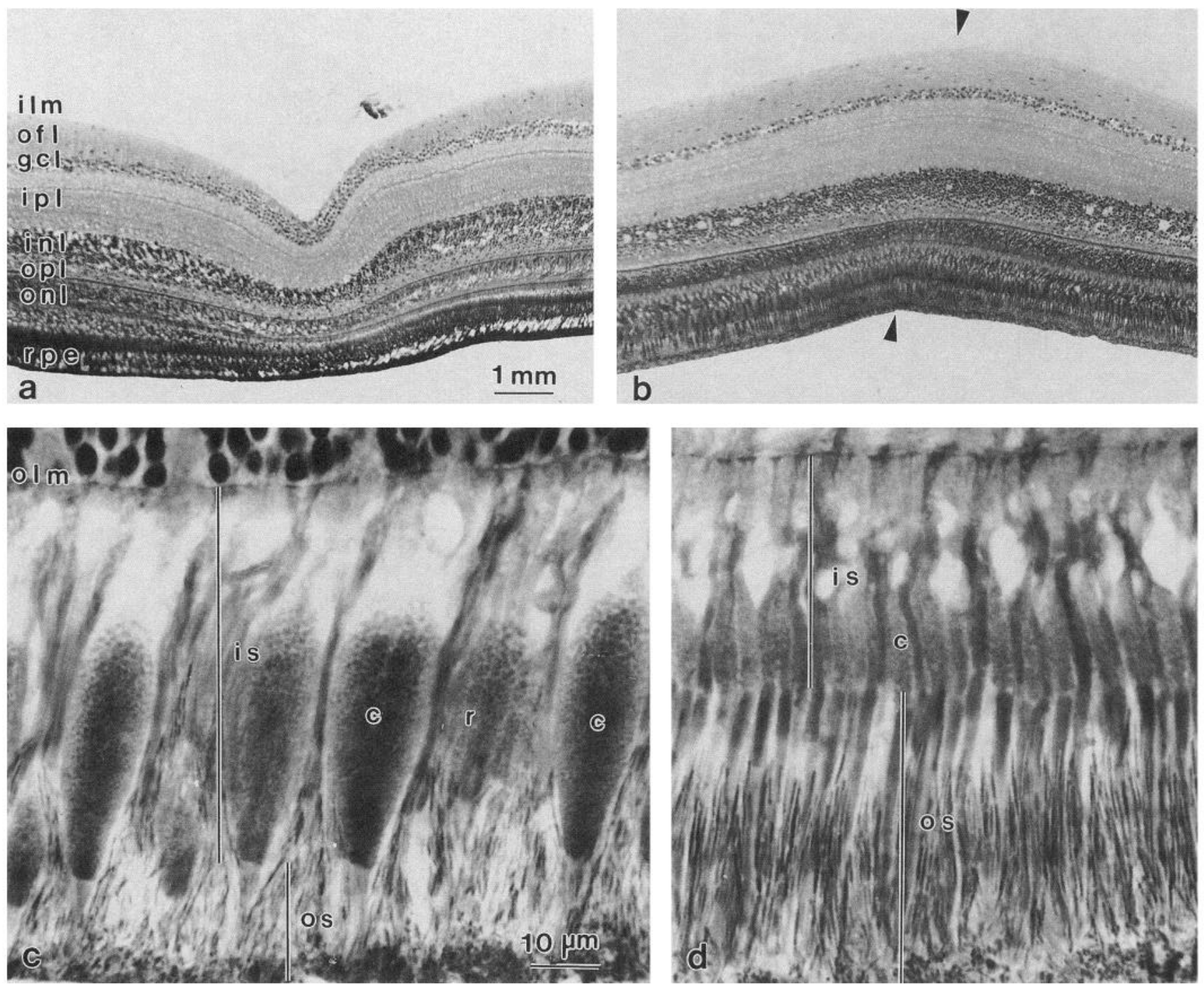

Figure 4. Histological sections of the retina of Parapercis nebulosa (Pinguipedidae) illustrating the fovea, the ridge, and variations in cone sizes and shapes. $a$, The fovea, cut along a dorsoventral plane (dorsal to the left). All the layers except the fiber layer are present in the pit, though all are deformed. $b$, The high-density horizontal ridge (between the two arrowheads). Densities in all layers are relatively high on the ridge, and photoreceptors are relatively small. $c$, The cone inner and outer segments several millimeters dorsal to the fovea. The cones are large and sparse, and have inner segments (denoted by vertical line labeled is) much longer than the outer segments (vertical lines labeled os). Rods are numerous, much smaller, and occupy the spaces between the cones. $d$, The same layer as in $c$, but adjacent to the fovea. The cones are thin and densely packed, and the cone outer segments are nearly as long as the inner segments. Rods can not be distinguished. $c$, cone; gcl, ganglion cell layer; $i l m$, inner limiting membrane; inl, inner nuclear layer; ipl, inner plexiform layer; is, inner segment of cone; ofl, optic fiber layer; olm, outer limiting membrane; onl, outer nuclear layer; opl, outer plexiform layer; os, outer segment of cone; $r$, rod; rpe, retinal pigmented epithelium. The scale bars in $a$ and $c$ apply to $b$ and $d$, respectively.

but differed from the pattern of Figure 1 in other respects. The blennies and goatfish had elongated optic papillas (see Fig. 9) and the pipefish had more complex fiber trajectories (see Fig. 8). These eight families are listed in Table 1 under "Symmetric growth."

\section{Asymmetric growth}

Figure 3 shows a right retina from a sand perch that was labeled through four sites in the nerve. The optic papilla is elongate and extends over much of the embryonic fissure, which is strongly curved. The fluorescent axons in the optic papilla are in four groups, each of which can be traced to an annulus of fluorescent cells, the outermost of which is relatively faint (compare Fig. $3 b, c)$.

Fovea. A fovea was recognized as a dimple on the inner limiting membrane, at the temporal end of the high-density band of cells noted by Collin and Pettigrew (1988b), and colinear with the central end of the optic papilla. Histological sections showed that all retinal layers, except that of the optic fibers, were present in the pit, although slightly compressed relative to neighboring regions (Fig. $4 a$ ). The exclusion of axons, potent scatterers of light, is ordinarily interpreted as a means of enhancement of the retinal image. Cell densities were much higher in the fovea than in neighboring regions, and the photoreceptors, predom- 

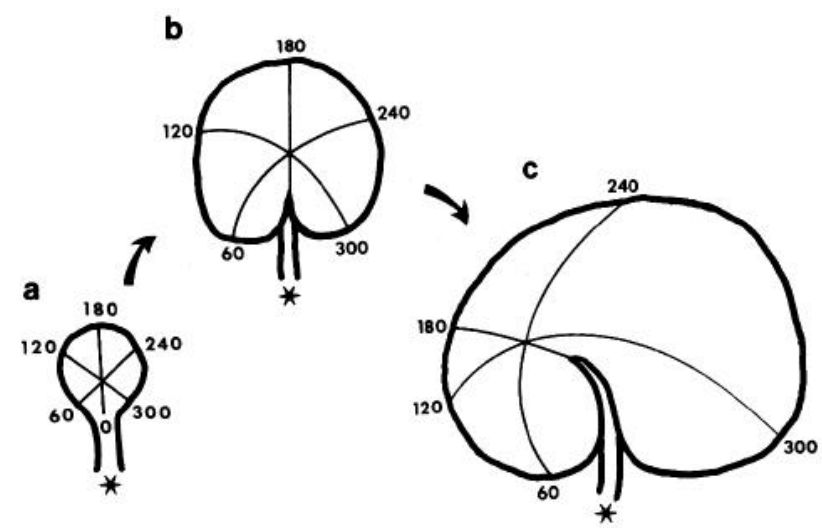

Figure 5. This is an interpretive drawing to illustrate three stages in the morphogenesis of a right retina such as the one in Figure 3. All three panels represent the retina, viewed en face. The numbers indicate the boundaries of the $60^{\circ}$ retinal sectors, referred to the coordinates of the early embryonic eye. $a$, This early retina, resembling a cobra's head, is a flattened, slightly concave surface at the end of the tubular optic stalk $(*)$. The oldest part of the retina lies near the intersection of the six rays. The ventral, temporal, dorsal, and nasal poles are at $0^{\circ}, 90^{\circ}, 180^{\circ}$, and $270^{\circ}$, respectively. $b$, As the retina enlarges, the growth on the ventral side is exaggerated, and the ventronasal and ventrotemporal quadrants extend downward to envelop the optic stalk, thereby forming the embryonic fissure. $c$, As the retina grows disproportionately on the nasal side (compare the $240-300^{\circ}$ sector to the $120-180^{\circ}$ sector), the oldest part (central retina in $a$ and $b$ ) comes to lie in the temporal part of the adult retina, the embryonic fissure bends temporally, and the $0 / 180^{\circ}$ ray, previously the dorsoventral indicator, is distorted to point nasotemporally.

inantly cones, were smaller in cross section (Fig. $4 c, d$ ). The absence of axons and the high densities and small dimensions of the cells, especially the photoreceptors, suggest that this fovea is specialized for high resolution. Most of the same morphological features were found in the horizontal strip of high cell density (Collin and Pettigrew, 1988b), but the axons were not displaced (Fig. $4 b$ ).

Annuli. The shapes and spacing of the annuli in Figure 3 have implications for growth. As in Figure 1, the nasal and temporal sides do not meet at the embryonic fissure. This separation has two causes. The first is a local one-the dorsal hook on the temporal cusp, mentioned above. The second is more globalthe space between adjacent labeled annuli is different on opposite sides of the fissure. This is true for all labeled annuli, but is most evident between the second and third, which are separated from one another by nearly twice as great a distance on the nasal side as on the temporal. This implies that the nasal side grew more between the times when the second and third annuli were established at the margin. This inequality had no doubt contributed to the curve of the embryonic fissure, analogous to the bending of a plant stem that results from more growth on the convex side. The space between adjacent annuli is smallest in the temporodorsal region, largest nasoventrally, and intermediate elsewhere. The consequence is that the innermost annulus, bounding the oldest part of retina, is in the temporal half of the adult retina. Moreover, the innermost annulus has rotated about $90^{\circ}$ relative to its original position. The central portion of the embryonic fissure, a dorsoventral structure originally, has curved to a temporodorsal orientation in the adult. If the orientation of the embryonic fissure is taken to be indicative of the dorsoventral axis at the time the nearby retina was being formed, then the original dorsoventral direction of the oldest retina has become temporonasal. In summary, this retina has grown asymmetrically, in a way that has favored the enlargement of the nasal half retina, and maintained the oldest part, containing the fovea, close to the temporal margin. The interpretive drawing of Figure 5 summarizes these events.

Axons. The axonal trajectories were quite complex. Inside the innermost annulus, the axons systematically coursed around, but not through, the fovea, consistent with the sections through the fovea (Fig. 4a). The circuitous axonal route was confirmed by directly labeling another sand perch retina at the margin opposite the central end of the optic papilla. As Figure 6 shows, the fibers did indeed avoid the area, reminiscent of the circumfoveal arcuate fibers in the human retina. This result suggests that the pattern of axon trajectories seen in Figures $3 a$ and 6 can be adopted as a diagnostic feature of an axon-free fovea.

The axons originating from the inner two annuli converge at the edges of these annuli, rather than at their centers, as in retinas with a central optic disk (compare Figs. 1, 3a). This eccentric exit presumably reflects the structure of the early optic cup, in which the dorsal retina, produced first, sent axons across its outer surface (the presumptive inner limiting membrane of the retina, or the pial surface of the CNS) and onto the ventral surface of the optic stalk, which was continuous with the ventral pole of the retina (Grant and Rubin, 1980; Holt, 1980). As was argued above, the nasal side of the inner annuli in the adult corresponds to the ventral side of the embryonic retina. As the retina enlarged, the new nasal and temporal surfaces enlarged in all directions, including ventrally, and opened like a fan around the optic stalk to encircle it, and thereby moving the ventral retinal pole considerably more ventral than the optic stalk (Fig.

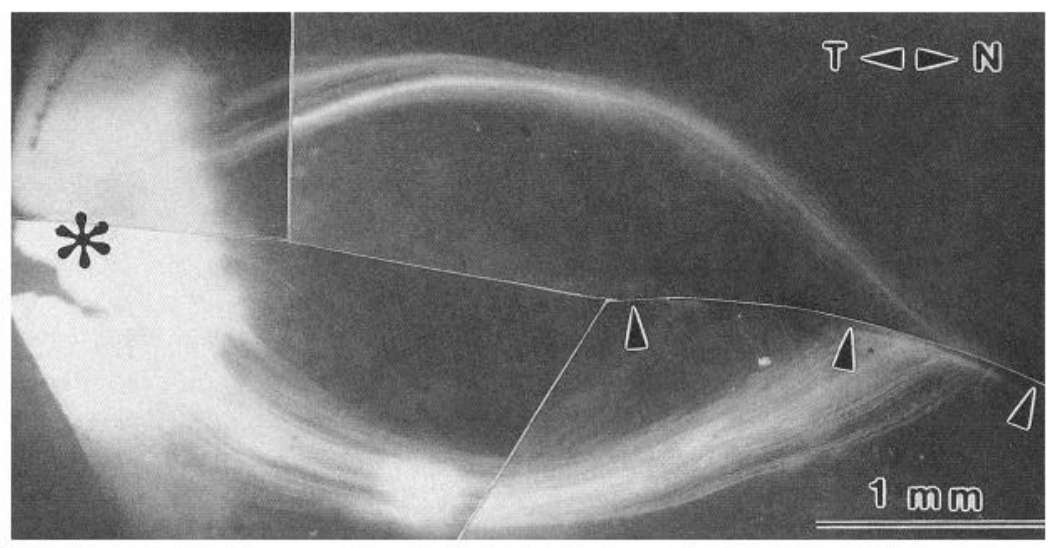

Figure 6. Right retinal whole-mount of Parapercis cylindrica showing skirting fibers. The DiI was applied at the retinal margin (asterisk), and the labeled fibers course around, not through, the fovea, to enter the optic papilla ( $a r$ rowheads). 


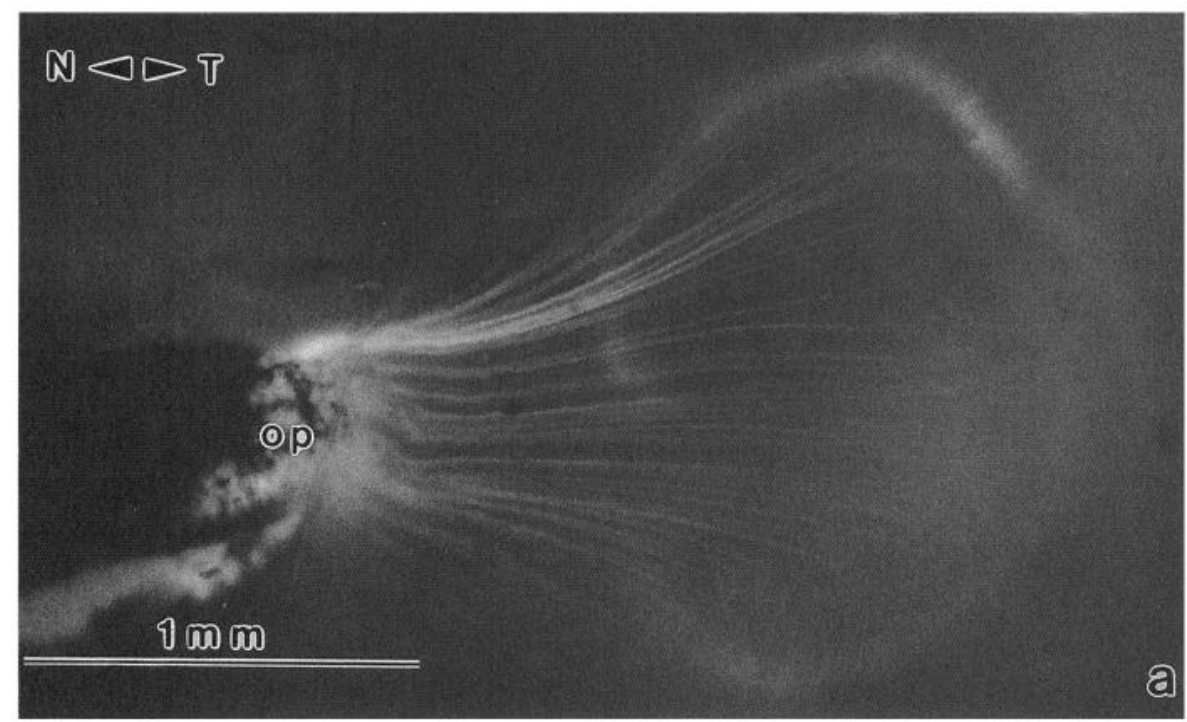

Figure 7. Left retinal whole-mount of Canthigaster valentini (Tetraodontidae) showing an early annulus of labeled cells displaced from the central end of the optic papilla $(o p)$. $a$, Low magnification. $b$, Higher magnification, illustrating the very eccentric exits of the axons.

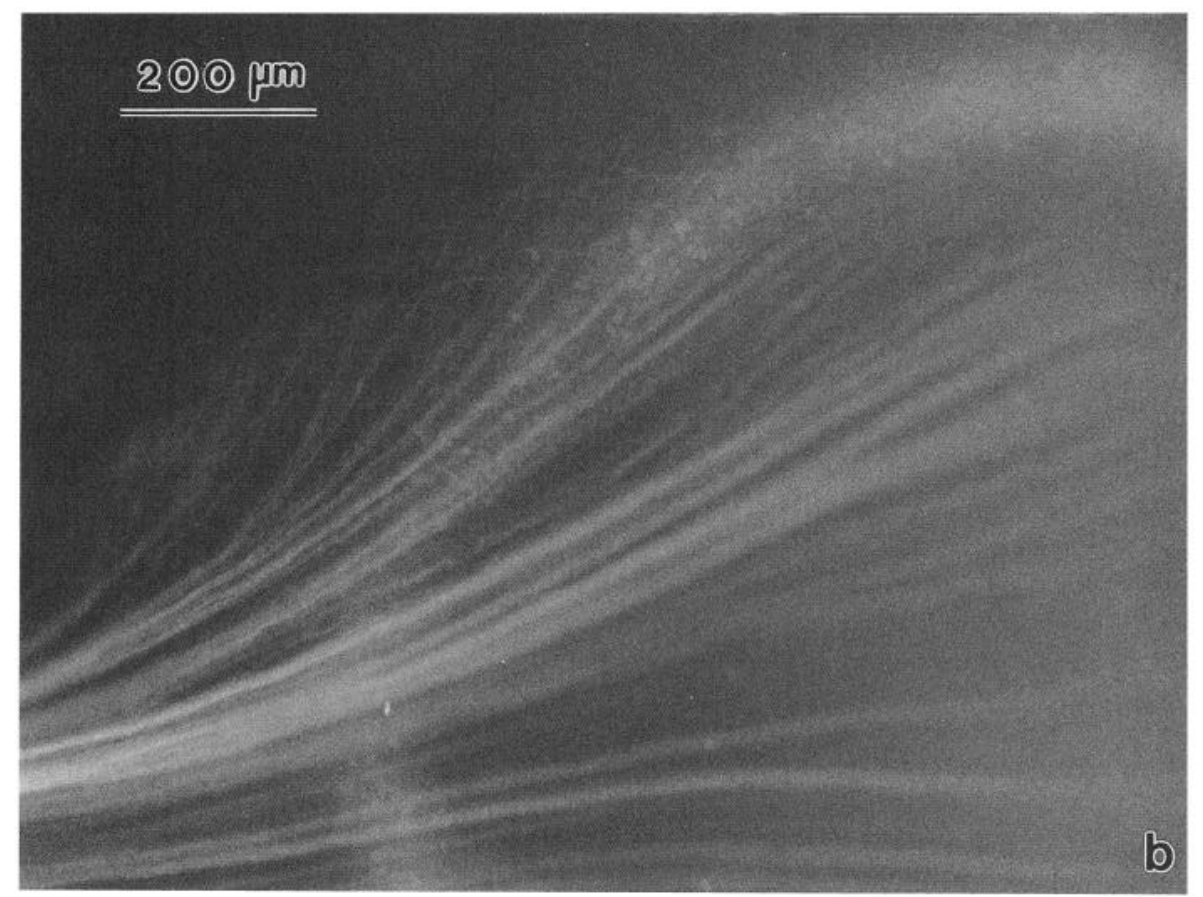

$5 a, b)$. Thus surrounded, the optic stalk receives axons from $360^{\circ}$.

The puffer fish and flathead retinas show an even more extreme case of eccentric exits by early fibers. Figure 7 illustrates that an early annulus is actually displaced from the adult optic papilla. The labeled somata presumably lay at the margin of an early eye cup from which the axons emerged ventrally onto the optic stalk (Fig. 5a). These fibers now course from that annulus across retina to the optic papilla, implying that the optic stalk gave rise to the part of the retina between the labeled annulus and the central end of the papilla.

Axons from different annuli were stratified in the optic fiber layer-the more peripheral their origin, the more superficial their axons (Scholes, 1979; Easter et al., 1984). Figure $3 d-f$ illustrates this point for axons passing over the same part of the retina. These images also show that axons had very different trajectories, depending on their origins. The superficial axons are oriented at nearly a right angle with respect to their deeper neighbors. This contrasts with the "spoke-on-a-wheel" arrangements in retinas with symmetric growth, in which all axons course parallel to their predecessors toward the center of the optic papilla (Fig. 1). In the retina in Figure 3, each new generation of axons is aimed toward a more ventral part of the optic papilla than its predecessors, and this forces a slightly different trajectory, which, cumulatively, produces the effect in Figure $3, e$ and $f$. This pathfinding appears more complex than the one faced by axons in Figure 1, but this impression may be illusory, as each generation of axons probably coursed along the route formed by their immediate predecessors (Scholes, 1979).

In summary, the sand perch retina is marked by (1) an optic papilla that curves temporally through nearly a right angle, (2) nonconcentric annuli that are close together on their temporal sides and more distant on the nasal sides, and (3) axons that have complex trajectories, including some that skirt the fovea. 

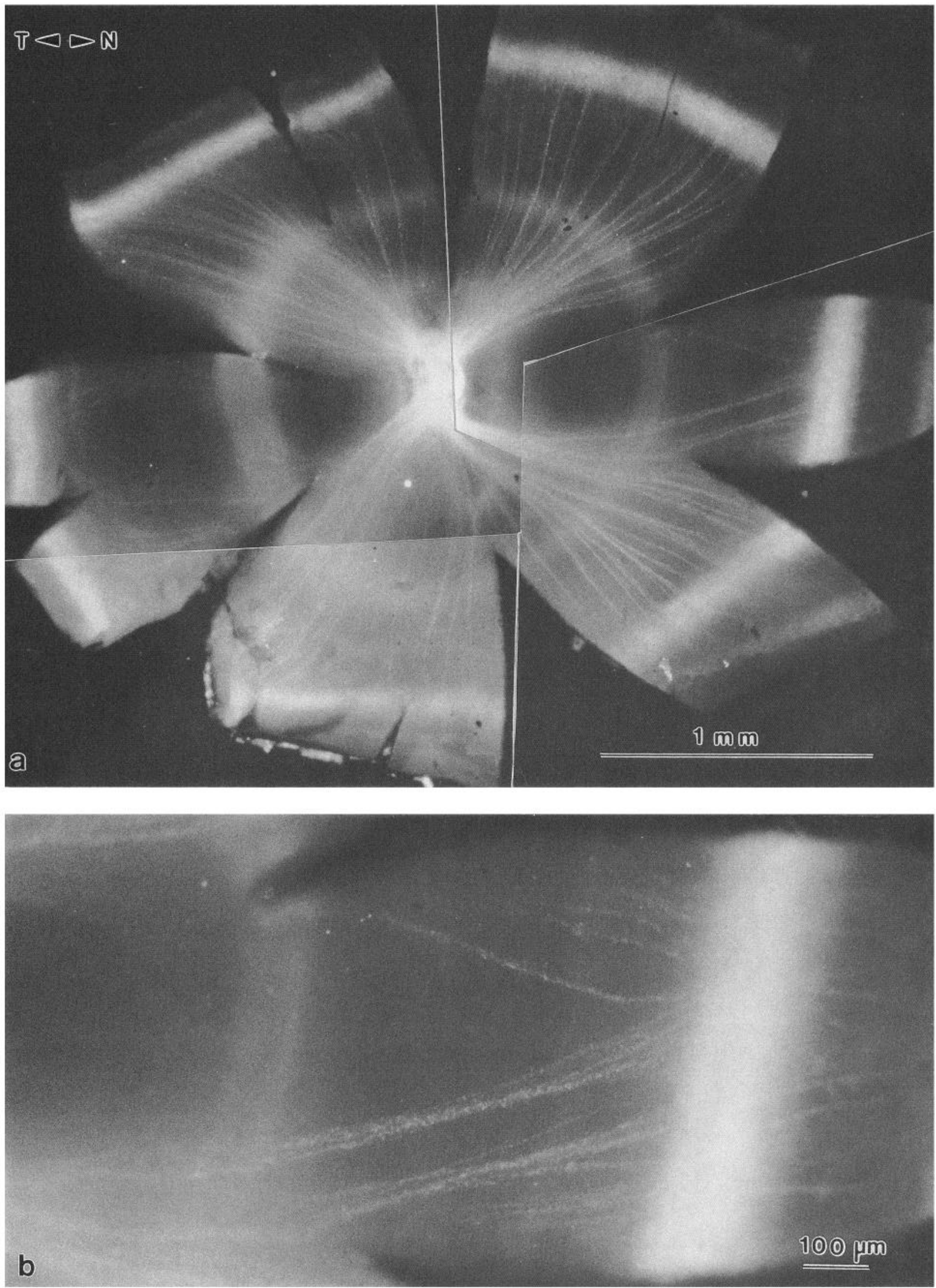

Figure 8. Right retinal whole-mount of Trachyrhamphus bioarctatus (Syngnathidae) showing symmetric growth and skirting fibers. a, Low magnification, showing symmetrical annuli. $b$, Detail of the skirting fibers on the nasal side. 


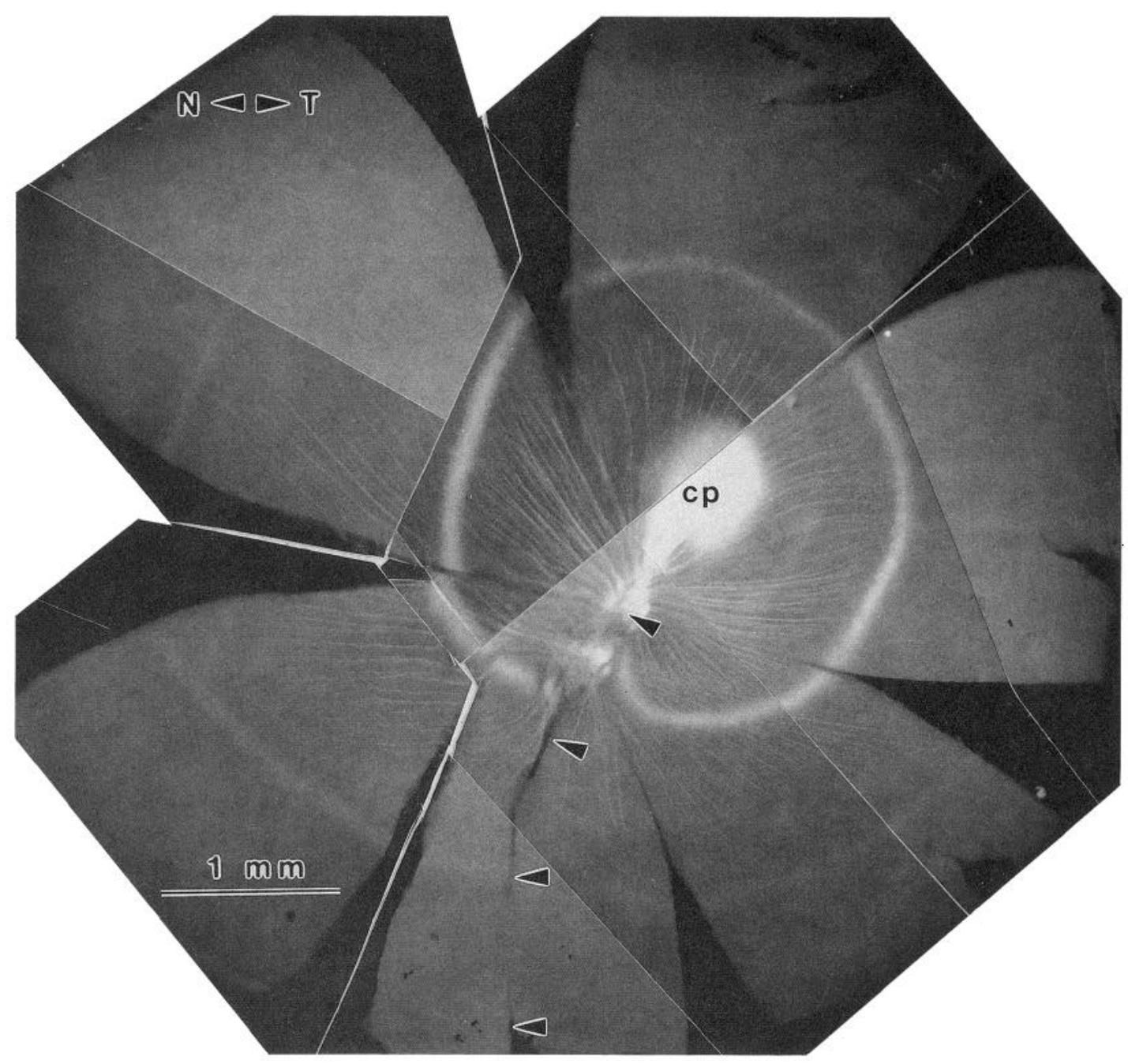

Figure 9. Left retinal whole-mount of Istriblennius edentula (Blenniidae) showing symmetrical retinal growth and no skirting fibers. A bright central patch of cells ( $c p$ ) dorsotemporal to the central end of the embryonic fissure (arrowheads) was labeled retrogradely through the nerve, as well as several annuli, all of which are approximately concentric with the central patch.

All three of these features were seen in the retinas of members of five other families: dragonets, hawkfish, leatherjackets, flatheads, and puffer fish, suggesting that all have axon-free foveas that have been maintained in the temporal retina by asymmetric growth. In the one wrasse that was examined, all characteristics except the skirting fibers were noted; the asymmetric growth suggests a specialized temporal area, probably an RCHD, but the absence of skirting fibers makes it unlikely to be a fovea. These seven families are listed in Table 1 under "Asymmetric growth."

These observations lead to the following generalization: the three morphological features are indicative of a foveated retina, or, failing the presence of skirting fibers, of a retina with a nonfoveal RHCD.

\section{Unexpected symmetric growth}

Some of the retinas that were anticipated to have specialized areas had not grown asymmetrically. As they seem to challenge the validity of the generalization drawn above, they are examined below.

Figure 8 shows a pipefish retina, in which Kahmann (1934) described a fovea. The optic papilla is nearly punctate, only slightly longer dorsoventrally than nasotemporally, and it lies at the approximate center of the retina. The labeled annuli are roughly concentric, and centered on the papilla. The axons that converge on it skirt around the temporonasal meridian on both sides of the papilla. The retinas of another species from this family (sea horse) were similar. Either these species lack an RHCD, contrary to the earlier report, or their growth pattern is inconsistent with the generalization summarized two paragraphs above.

The presence of an RHCD was checked by sectioning both retinas from one sea horse and saving all sections. They showed that the temporal hemiretina was considerably thicker, and had higher cell densities, than the nasal hemiretina. However, neither side had a fovea, or even a local RHCD, and given that the axons were very sparse in most of the middle of the retina (Fig. 8), there was no possibility of a fovea sculpted from the fiber layer, as in Figure $4 a$. I conclude that the pipefish/sea horse family is not an exception to the generalization that fish retinas with an RHCD grow asymmetrically.

Figure 9 shows a retina from a blenny, a family with some foveated species (Kahmann, 1934). In contrast to the retinas that had grown asymmetrically, the optic papilla is rather short, 
the embryonic fissure is nearly straight, and the labeled annuli are approximately concentric with the bright central patch of labeled cells. Moreover, the axons converge directly, without skirting any area, toward exits inside the annuli. The retina of the other blenny that was examined was very similar, as were the retinas of two damselfish species. Thus, they did not fit the generalization, and reasons for this will be offered in the Discussion.

\section{Discussion}

\section{Life-style and RHCD}

Why some fish have RHCDs and others do not is not known. Walls (1942) suggested that only those animals with a specialized retinal region "look at" objects, that is, shift their eyes so as to bring the object of interest on to the specialized region. This seems a reasonable interpretation, but an adaptive link between life-style and retinal structure remains elusive. Of the seven families that were inferred to have RHCDs, four (dragonets, hawkfish, sand perch, and flatheads) spend most of their time relatively motionless. Many of the sea basses (Serranidae) that have foveas (Schwassmann, 1968) are similarly motionless, so it might be thought that a stationary platform for the eyes was a prerequisite for possession of an RHCD. However, the blennies and the jawfish are also motionless and lack the RHCD, and the puffer fish, wrasses, and leatherjackets, all of which move around the water column quite actively, have the RHCD. The answer remains obscure.

\section{Foveal development in fish and mammals}

The "incomplete" fovea, with all other layers except the axons present, is structurally very different from the "complete" toveas in various reptilian, avian, and mammalian species, in which all but the receptors are pushed aside (Kahmann, 1936). However, the developmental origin of the specialized area in the seven teleost families exemplified by the sand perch (Fig. 3) resembles in one respect the origin of analogous mammalian structures. The oldest part of the adult mammalian retina is typically at or near the RHCD (reviewed by Robinson, 1991), as in the asymmetrically growing retinas described above. Hendrickson and her collaborators (Yuodelis and Hendrickson, 1986; Packer et al., 1990) have shown that the primate fovea takes shape over a prolonged period, during which a locally high density of cones becomes even higher as surrounding cones migrate into the central retina, and the pit is sculpted out by the migration of interneurons toward the periphery. The formation of the fish fovea must have differed, as it did not involve the emigration of interneurons. The density of cones may or may not have changed. Planimetric densities of all neurons are highest in the retinas of young fish (Johns and Eastcr, 1977), so if the earliest retina stretched less than the retina that was subsequently added at its margin, a central RHCD would result, and immigration of other cells would accentuate this peak.

Later in development, the differences between fish and mammals are more striking than the similarities. In mammals with frontal eyes, an RHCD in central retina will point anteriorly, and approximately symmetric retinal growth will maintain that orientation. In contrast, fish have lateral eyes, and so require asymmetric growth if the early retina is to occupy a temporal position. A second difference is in the relation of retinal growth and life span. In mammals, retinal histogenesis is virtually complete by the time the animal begins to use its eyes. In fish, most retinal growth occurs after hatching, while the eyes are in use.
Thus, the formation of the fovea is constrained by functional considerations not present in mammals.

\section{Apparent failures of the generalization}

If asymmetrical retinal growth will provide for the maintenance of an RHCD in temporal retina, what of the other retinas, from blennies and damselfish, that were suspected of having an RHCD but were found to grow symmetrically?

Collin (1987) described a dorsotemporal RHCD in Istiblennius edentula, the same species shown in Figure 9, and the ganglion cell density in that region was only about twice that of the lowest density elsewhere in the same retina. An even smaller difference between maximum and minimum was found in the one damselfish retina that he described (Amblyglyphidodon $\mathrm{Cu}$ racao). Such small variations, comparable to those in the goldfish (Mednick and Springer, 1988), may not be significant, either functionally or statistically - they may lie within the range of normal variation of density across the entire retina. It would be useful to know how reproducible the estimates of cell density are on the contralateral retinas of the individuals described, and on other conspecifics. I suggest that the blennies and damselfish in question did not show asymmetric growth because the RHCDs are probably not important functionally.

The pipefish/sea horse family was claimed to have foveas (Kahmann, 1934), and they have skirting fibers (Fig. 8), yet they grew symmetrically. Our histology showed that their retinas did not have foveas and that the RHCD was actually an entire hemiretina. It is unclear why Kahmann (1934) concluded that they had a fovea. He examined a different species than ours, and perhaps that explains the difference, but the photomicrograph that he offers (his Fig. $3 a$ ) is not a convincing image of a fovea. In his other work (e.g., Kahmann, 1936, Fig. 2), he shows that he has a very low threshold for calling a structure a fovea. Alternatively, he may have been misled by examining dorsoventral sections, which would have fewer axons centrally than peripherally, owing to the skirting fibers (Fig. 8).

\section{Summary and conclusions}

Asymmetric retinal growth can be understood as a solution to a particular visual problem. The mechanism probably involves one or all of three regionally variable processes: cell proliferation, cell death, and retinal stretch. In addition, the existence of a patent embryonic fissure enables the two halves of the retina to slide with respect to one another; indeed, this sliding may be the event that causes the fissure to remain patent rather than fused as it does in other vertebrates. The control of this complex pattern of retinal growth-experiential or programmed without reference to experience-remains unknown.

\section{References}

Anders JJ, Hibbard E (1974) The optic system of the teleost Cichlasoma biocellatum. J Comp Neurol 158:145-154.

Collin SP (1987) Retinal topography in reef teleosts. PhD dissertation, University of Queensland, Brisbane, Australia.

Collin SP, Pettigrew JD (1988a) Retinal topography in reef teleosts. I. Some species with well-developed areae but poorly-developed streaks. Brain Behav Evol 31:269-282.

Collin SP, Pettigrew JD (1988b) Retinal topography in reef teleosts. II. Some species with prominent horizontal streaks and high-density areae. Brain Behav Evol 31:283-295.

Collin SP, Pettigrew JD (1989) Quantitative comparison of the limits on visual spatial resolution set by the ganglion cell layer in twelve species of reef teleosts. Brain Behav Evol 34:184-192. 
Easter SS Jr (1991) Radially asymmetric growth of fish retinas with specialized areas. Soc Neurosci Abstr 17:560.

Easter SS Jr, Johns PR, Baumann LR (1977) Growth of the adult goldfish eye I. Optics. Vision Res 15:469-477.

Easter SS Jr, Rusoff AC, Kish PE (1981) The growth and organization of the optic nerve and tract in juvenile and adult goldfish. J Neurosci 1:793-811.

Easter SS Jr, Bratton B, Scherer SS (1984) Growth-related order in the retinal fiber layer in goldfish. J Neurosci 4:2173-2190.

Godement $P$, Vanselow J, Thanos S, Bonhoeffer $F$ (1987) A study in developing visual systems with a new method of staining neurones and their processes in fixed tissue. Development 101:697-713.

Grant P, Rubin E (1980) Ontogeny of the retina and optic nerve in Xenopus laevis. II. Ontogeny of the optic fiber pattern in the retina. J Comp Neurol 189:671-698

Hollyfield J (1972) Histogenesis of the retina of the killifish Fundulus heteroclitus. J Comp Neurol 144:373-388.

Holt C (1980) Cell movements in Xenopus eye development. Nature $287: 850-852$.

Honig MG, Hume RI (1986) Fluorescent carbocyanine dyes allow living neurones of identified origin to be studied in long-term cultures. J Cell Biol 103:171-187.

Johns PR (1977) Growth of the adult goldfish eye. III. Source of the new retinal cells. J Comp Neurol 176:343-358.

Johns PR, Easter SS Jr (1977) Growth of the adult goldfish eye. II. Increase in retinal cell number. J Comp Neurol 176:331-342.

Johns PR, Fernald RD (1981) Genesis of rods in teleost fish retina. Nature 293:141-142.

Kahmann H (1934) Ueber die Vorkommen einer Fovea centralis im Knochenfischauge. Zool Anz 106:49-55.

Kahmann H (1936) Ueber das foveale Sehen der Wirbeltiere. I. Ueber die Fovea centralis und die Fovea lateralis bei einigen Wirbeltieren. Arch f Ophthalmol (Copenh) 135:265-276.
Mednik AS, Springer AD (1988) Asymmetric distribution of retinal ganglion cells in goldfish. J Comp Neurol 268:49-59.

Meyer RL (1978) Evidence from thymidine labelling for continuing growth of retina and tectum in juvenile goldfish. Exp Neurol 59:99111.

Muller H (1952) Bau und Wachstum der Netzhaut des Guppy (Lebistes reticulatus). Zool Jahrb 63:275-324.

Packer O, Hendrickson AE, Curcio CA (1990) Developmental redistribution of photoreceptors across the Macaca nemestrina (pigtail macaque) retina. J Comp Neurol 298:472-493.

Randall JE, Allen GR, Steene RC (1990) Fishes of the Great Barrier Reef and Coral Sea. Bathurst: Crawford.

Raymond PR, Rivlin PK (1987) Germinal cells in the goldfish retina that produce rod photoreceptors. Dev Biol 122:120-138.

Robinson SR (1991) Development of the mammalian retina. In: The neuroanatomy of the visual pathways and their development (Dreher B, Robinson S, eds), pp 69-128. London: Macmillan.

Rusoff AC, Easter SS Jr (1980) Order in the optic nerve of goldfish. Science 208:311-312.

Scholes JH (1979) Nerve fibre topography in the retinal projection to the tectum. Nature 278:620-624.

Schwassmann HO (1968) Visual projections upon the tectum in foveate marine teleosts. Vision Res 8:1337-1348.

Tapp RL (1973) The structure of the optic nerve of the teleost, Eugerres plumieri. J Comp Neurol 150:239-252.

Walls $G$ (1942) The vertebrate eye and its adaptive radiation. New York: Hafner.

Yuodelis C, Hendrickson A (1986) A qualitative and quantitative analysis of the human fovea during development. Vision Res $26: 847-$ 855. 